August 22, 2018

\title{
The nucleon axial mass and the MiniBooNE Quasielastic Neutrino-Nucleus Scattering problem
}

\author{
J. Nieves, ${ }^{1}$ I. Ruiz Simo, ${ }^{2}$ and M. J. Vicente Vacas $^{2}$ \\ ${ }^{1}$ Instituto de Física Corpuscular (IFIC), Centro Mixto Universidad de Valencia-CSIC, \\ Institutos de Investigación de Paterna, E-46071 Valencia, Spain \\ ${ }^{2}$ Departamento de Fúsica Teórica and IFIC, Centro Mixto Universidad de Valencia-CSIC, \\ Institutos de Investigación de Paterna, E-46071 Valencia, Spain
}

\begin{abstract}
The charged-current double differential neutrino cross section, measured by the MiniBooNE Collaboration, has been analyzed using a microscopical model that accounts for, among other nuclear effects, long range nuclear (RPA) correlations and multinucleon scattering. We find that MiniBooNE data are fully compatible with the world average of the nucleon axial mass in contrast with several previous analyses which have suggested an anomalously large value. We also discuss the reliability of the algorithm used to estimate the neutrino energy.

PACS numbers: 25.30.Pt,13.15.+g, 24.10.Cn,21.60.Jz
\end{abstract}

Elastic neutrino nucleon scattering can be described by three dominant form factors. The two vector form factors $F_{1,2}\left(Q^{2}\right)$ are well known from electron scattering (see, e.g. [1], for a review). The axial-vector form factor at $Q^{2}=0$, $F_{A}(0)$, is determined from neutron $\beta$ decay. Assuming a dipole form, the $Q^{2}$ dependence of $F_{A}=F_{A}(0)\left(1+Q^{2} / M_{A}^{2}\right)^{-2}$ can be characterized by the axial mass $M_{A}$. The value $M_{A}=1.03 \pm 0.02 \mathrm{GeV}$ is usually quoted as the world average [2, 3], although a recent analysis claims an even smaller uncertainty $\left(M_{A}=1.014 \pm 0.014\right.$ [4] $)$. It should be remarked that there are two independent experimental sources of information for this parameter, neutrino/antineutrino induced reactions and pion electroproduction. In the first case, bubble chamber data for $\nu$-deuterium quasielastic (QE) scattering play a dominant role. The initial apparent disagreement between the values of $M_{A}$ obtained with weak and electromagnetic probes was solved after correcting for hadronic effects [2] and now both sets of data are consistent. With these ingredients it looked straightforward to describe $\nu$ QE scattering in nuclei with the high precision required by the new and forthcoming neutrino experiments, that aim to measure parameters such as the $\theta_{13}$ mixing angle or the leptonic $\mathrm{CP}$ violation.

In this context, the charged current QE MiniBooNE data [5] have been quite surprising. First, the absolute values of the cross section are too large as compared to the consensus of theoretical models [6, 7]. Actually, the cross section per nucleon on ${ }^{12} C$ is clearly larger than for free nucleons. Second, their fit to the shape (excluding normalization) of the $Q^{2}$ distribution leads to an axial mass, $M_{A}=1.35 \pm 0.17 \mathrm{GeV}$, much larger than the previous world average. In fact, the large value of $M_{A}$ also implies a substantial increase in the total cross section predicted by the Relativistic Fermi Gas model used in the analysis, improving the agreement with the size of the cross section.

Similar results have been later obtained analyzing MiniBooNE data with more sophisticated treatments of the nuclear effects that work well in the study of electron scattering. For instance, Refs. [8, 9] using the impulse approximation with state of the art spectral functions for the nucleons fail to reproduce data with standard values of $M_{A}$. Large axial mass values have also been obtained in Ref. [10] in a Fermi gas model and using spectral functions and in Ref. [1] , where data have been analyzed in a relativistic distorted-wave impulse approximation and with a relativistic Fermi gas model.

Certainly, there are some caveats that should be kept in mind like the flux uncertainty or inadequacies on the subtraction of background processes such as pion production. However, the associated uncertainties have been estimated and included in the error bands provided in Ref. [5] and in the previously quoted analyses. Nonetheless, being the axial mass relatively well established by electron data, the failure to describe the MiniBooNE data with standard values of $M_{A}$ could point out more to the incompleteness of the theoretical models than to the need of reconsidering the value of the parameter.

As a consequence, several approaches incorporating new mechanisms that could contribute to the QE signal have been explored. An important step was undertaken in Refs. 12, 13] with the inclusion of two nucleon mechanisms and other multinucleon excitations related to the $\Delta$ resonance. These works could reproduce the MiniBooNE total QE cross section without modifying the axial mass, suggesting that a good part of the experimental cross section was not strictly QE scattering. The importance of meson exchange currents and multinucleon excitations has also been explored in Ref. [14]. A microscopic model for two nucleon excitation and pion production was studied in Ref. [15], supporting the findings of Refs. [12, 13]. This latter model was a natural extension of the work in Refs. [16 18], where the purely quasielastic contribution to the inclusive electron and neutrino scattering on nuclei had been analyzed. The model includes one, two, and even three-nucleon mechanisms, as well as the excitation of $\Delta$ isobars. There are 
no free parameters in the description of nuclear effects, since they were fixed in previous studies of photon, electron, and pion interactions with nuclei 18 23].

In Refs. [12, 13, 15] only the total cross section was evaluated and compared with the so called "unfolded" data of Ref. [5]. Certainly, the experimental data include energy and angle distributions and therefore provide a much richer information. Furthermore, the unfolded cross section is not a very clean observable after noticing the importance of multinucleon mechanisms, because the unfolding itself is model dependent and assumes that the events are purely QE. The same limitation occurs for the differential cross section $d \sigma / d Q^{2}$, given that $Q^{2}$ is also deduced assuming the events are QE. From that point of view, the best observable to compare with theoretical models, and possibly constrain parameters, is the double differential cross section $d^{2} \sigma / d T_{\mu} d \cos \theta_{\mu}$ because both the muon angle and energy are directly measured quantities. Our aim in this work, is to analyse this latter observable within the theoretical model of Refs. [15 17]. Full details of the approach can be found there.

Here, we will briefly recall the main features of the model. It starts from a relativistic local Fermi gas (LFG) picture of the nucleus, which automatically accounts for Pauli blocking and Fermi motion. The QE contribution was studied in Ref. [16] incorporating several nuclear effects. The main one is the medium polarization (RPA), including $\Delta$-hole degrees of freedom and explicit $\pi$ and $\rho$ meson exchanges in the vector-isovector channel of the effective nucleonnucleon interaction. A correct energy balance is imposed using the experimental Q values. We will use here the full relativistic model of Ref. [16] without the inclusion of FSI interaction. The reason is that FSI was implemented in a nonrelativistic approach that makes it unsuitable for the large momenta transferred that are reached in the experiment under study. As it was discussed in [16], these FSI effects are always smaller than 7 percent for the total cross section ${ }^{1}$ but could be more important in the angle and energy distributions for the low neutrino energies studied in [16]. In Ref. [9], it was found that the main effect of FSI is a shift of $\sim 10 \mathrm{MeV}$ of the QE peak for neutrino energies closer to the MiniBooNE neutrino flux mean energy, $\left\langle E_{\nu}\right\rangle \sim 800 \mathrm{MeV}$, although that could depend on details of the model [24].

The model for multinucleon mechanisms (not properly QE but included in the MiniBooNE data [5]) has been fully discussed in Ref. [15]. It contains some additional uncertainty sources related to the detailed model for nucleon nucleon correlations, the $\Delta$ axial couplings [15] or the use of inconsistent treatment of its vertices and propagator [25].

To estimate the quality of the fit we use the following definition of $\chi^{2}$ that properly takes into account the global normalization uncertainty $(\Delta \lambda=0.107)$ following the procedure of $[26$,

$$
\chi^{2}=\sum_{i=1}^{137}\left[\frac{\lambda\left(\frac{d^{2} \sigma^{e x p}}{d T_{\mu} d \cos \theta}\right)_{i}-\left(\frac{d^{2} \sigma^{t h}}{d T_{\mu} d \cos \theta}\right)_{i}}{\lambda \Delta\left(\frac{d^{2} \sigma}{d T_{\mu} d \cos \theta}\right)_{i}}\right]^{2}+\left(\frac{\lambda-1}{\Delta \lambda}\right)^{2},
$$

where $\lambda$ is a global scale. $\frac{d^{2} \sigma^{e x p}}{d T_{\mu} d \cos \theta}$ is the experimental cross section and $\Delta\left(\frac{d^{2} \sigma}{d T_{\mu} d \cos \theta}\right)$ its uncertainty, both taken from Ref. [5]. The sum runs over the 137 angle-energy bins with a cross section different from zero in Ref. [5].

As a first test, we have minimized $\chi^{2}$ as a function of the axial mass in a simplified version of the model without multinucleon mechanisms and without RPA. This model should be quite similar to the one originally used in the MiniBooNE analysis. The main difference being that we use a local rather than global Fermi gas in the calculation. We obtain $M_{A}=1.32 \pm 0.03 \mathrm{GeV}$ with $\chi^{2}=35$. The fit is obviously very good and in agreement with Ref. [5]. The fitted scale is $\lambda=0.96 \pm 0.03$ also supporting MiniBooNE findings, that a shape-only fit was also consistent with the total cross section.

As a second test, we consider the full model with the same axial mass used in our previous papers $\left(M_{A}=1.049 \mathrm{GeV}\right)$. The results corresponding to these two versions of the model are shown in Fig. 1, The full model also agrees remarkably well with data. For this case we have $\chi^{2}=52$ with $\lambda=0.89 \pm 0.01$. This could look much worse, but it is still a very good agreement with $\chi^{2}$ per degree of freedom much lower than one and obtained without fitting any parameter of the theoretical model. Furthermore, the shape is very good and $\chi^{2}$ strongly depends on the normalization (scale and axial mass are strongly correlated). Therefore, from the quality of the fit only, one could not discriminate between the two versions of the model. However, we should recall that the RPA correlations and multinucleon mechanisms correspond to real nuclear effects that must be incorporated in the models. Although, we think the consistency of MiniBooNE data with standard values of $M_{A}$ has been established now, one could still go further and use our full model to fit the data letting $M_{A}$ to be a free parameter. We get $M_{A}=1.077 \pm 0.027 \mathrm{GeV}$ and $\lambda=0.917 \pm 0.029$ with a strong correlation between both parameters. For this case, $\chi^{2}=50$. The 1 and $2 \sigma$ contours are plotted in Fig. 2, This is a somewhat large value for $M_{A}$ but we think, the uncertainty size could be grossly underestimated. Notice first that, in the absence of a proper correlation matrix, the experimental uncertainties, except for the normalization,

1 Thus, the results for the total cross section without FSI are still inside the uncertainty band of Ref. [15] 


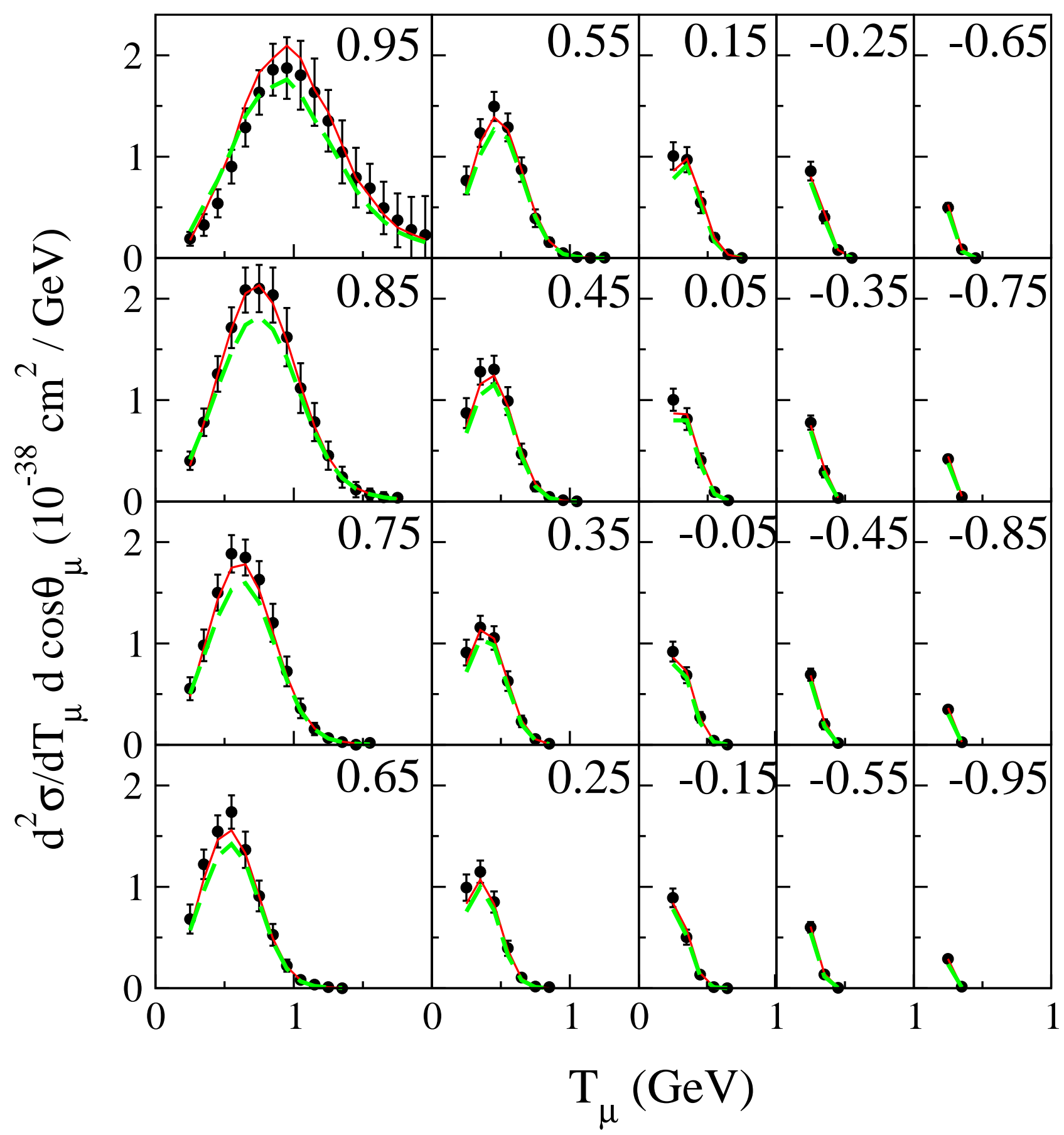

FIG. 1: Muon angle and energy distribution $d^{2} \sigma / d \cos \theta_{\mu} d T_{\mu}$. Different panels correspond to the various angular bins labeled by their cosinus central value. Experimental points from Ref. [5]. Green-dashed line (no fit) is the full model (including multinucleon mechanisms and RPA) and calculated with $M_{A}=1.049 \mathrm{GeV}$. Red-solid line is best fit $\left(M_{A}=1.32 \mathrm{GeV}\right)$ for the model without RPA and without multinucleon mechanisms.

have been treated as fully uncorrelated. In addition, one should include in the minimization procedure not only the experimental but also the theoretical uncertainties related to other parameters of the model (e.g. $\pi N N$ form factors, short range correlations, $\Delta$ in medium selfenergies, etc.).

The consideration of RPA and multinucleon mechanisms makes the present model more appropriate than a pure impulse approximation for the low momentum transfer region. Nonetheless, at very low momenta a more detailed treatment of the nuclear degrees of freedom could be necessary. As done in Ref. [10], we could exclude from the analysis the bins with a large contribution of small momentum transfer. There is some arbitrariness in the actual 


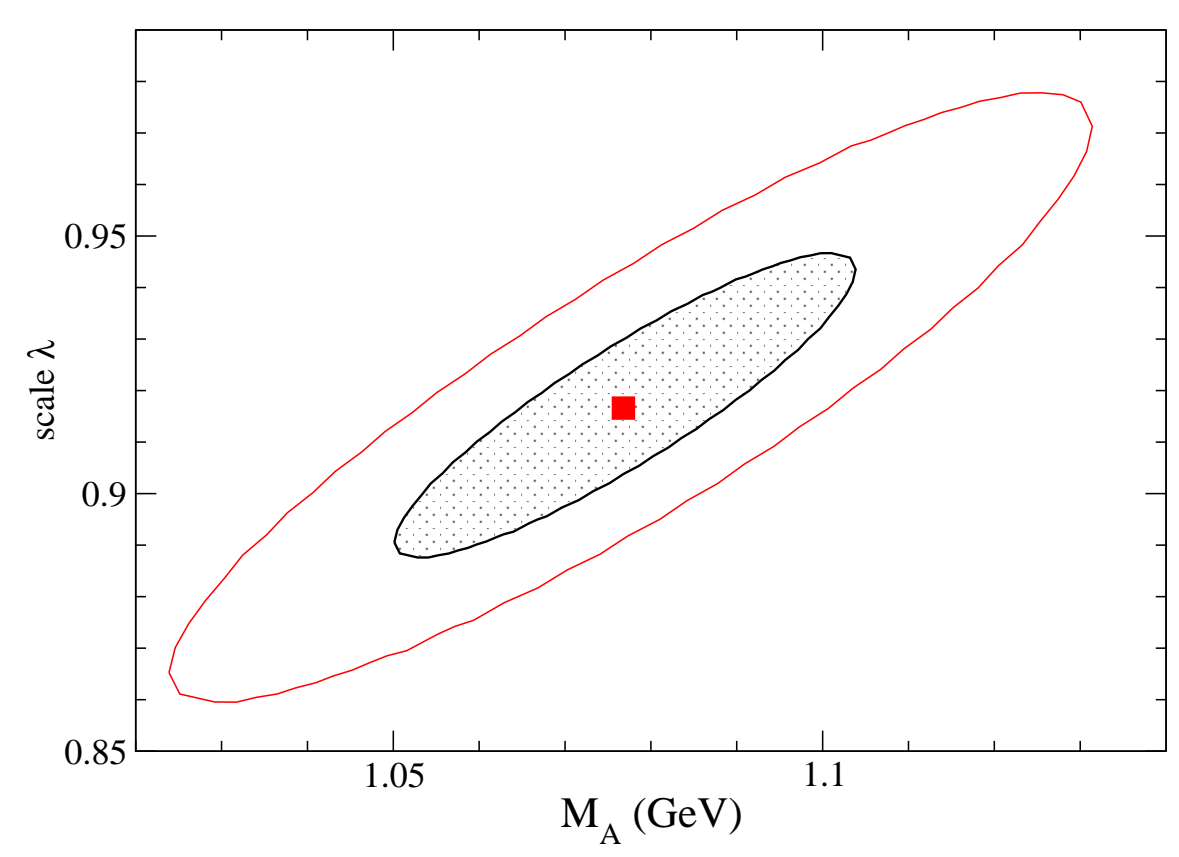

FIG. 2: Best fit value of $M_{A}$ and $\lambda$ and 1 and $2 \sigma$ regions.

TABLE I: Fit results for various models. See description in the text.

\begin{tabular}{|l||r|r|r|}
\hline Model & Scale & $M_{A}(\mathrm{GeV})$ & $\chi^{2} / \#$ bins \\
\hline LFG & $0.96 \pm 0.03$ & $1.321 \pm 0.030$ & $33 / 137$ \\
\hline Full & $0.92 \pm 0.03$ & $1.077 \pm 0.027$ & $50 / 137$ \\
\hline Full, $q_{\text {cut }}=400 \mathrm{MeV}$ & $0.83 \pm 0.04$ & $1.007 \pm 0.034$ & $30 / 123$ \\
\hline
\end{tabular}

choice of the cuts, but to allow for an easy comparison we have followed the procedure of Ref. [10] and implemented a transfer momentum threshold $q_{c u t}=400 \mathrm{MeV}$. This eliminates 14 of the 137 measured bins (see Fig. 3 from [10]). The fitted axial mass is then reduced to $M_{A}=1.007 \pm 0.034 \mathrm{GeV}$ and $\lambda=0.83 \pm 0.04$. As it is the case for the full calculation, the inclusion of multinucleon mechanisms and RPA is essential to obtain axial masses consistent with the world average. For all cases the best agreement is obtained for scale values lower than one. This is even clearer for standard values of $M_{A}$. Whereas this possible overestimation of the cross section could come from various sources the simplest explanation is some underestimation of the neutrino flux.

Finally, in Fig. 3, we show the contribution of the various mechanisms to the differential cross section at $0.80<$ $\cos \theta_{\mu}<0.90$. The experimental data have been scaled to help in the discussion. The results of the LFG model (without RPA or multinucleon effects) with a large $M_{A}(=1.32)$ and with the same scale as data clearly provide an excellent fit, as it has been found by other groups. For the rest of the curves we have taken $M_{A}=1.049 \mathrm{GeV}$ as in our previous papers 15 17]. The LFG model with the low value of $M_{A}$, allowing for a 10 percent normalization uncertainty, also provides an acceptable description of the data. One should remark that whereas this simple model agrees well for low and medium muon energies, it is systematically below data at high energies. The inclusion of collective effects (RPA), dotted line, slightly improves the agreement at these high energies. However, RPA strongly decreases the cross section at low energies. Multinucleon mechanisms, which in average get a larger energy transferred and thus accumulate their contribution at low muon energies compensate that depletion. Therefore, the final picture for this observable is that of a delicate balance between a dominant single nucleon scattering, corrected by collective effects, and other mechanisms that involve directly two or more nucleons. As shown, both effects can be mimicked by using a large $M_{A}$ value. It is also clear from this figure, that the proportion of multinucleon events contributing to the "QE" signal is quite large for low muon energies and thus, the algorithm commonly used to reconstruct the neutrino energy is badly suited for this region. This could have serious consequences in the determination of the oscillation parameters (see, e.g., discussion in Ref. [27] and Ref. [28]).

In summary, we have analyzed the MiniBooNE CCQE double differential cross-section data using the theoretical model of Refs. 15 17]. The model, that starts from a relativistic local Fermi gas description of the nucleus, includes RPA correlations and multinucleon effects. The same model is quite successful in the analysis of nuclear reactions 


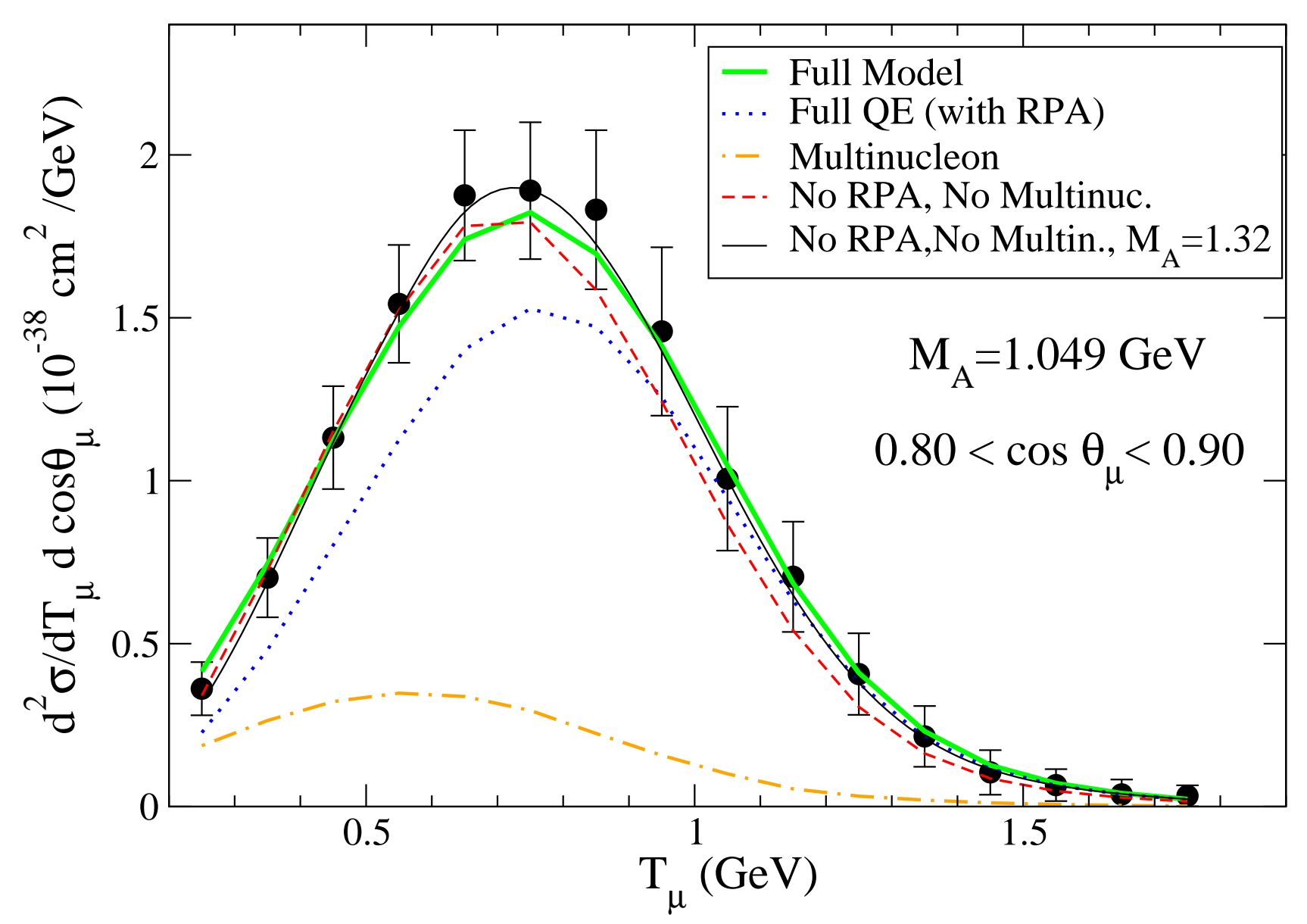

FIG. 3: Muon angle and energy distribution $d^{2} \sigma / d \cos \theta_{\mu} d T_{\mu}$ for $0.80<\cos \theta_{\mu}<0.90$. Experimental data from Ref. [5] and calculation with $M_{A}=1.32 \mathrm{GeV}$ are multiplied by 0.9 . Axial mass for the other curves is $M_{A}=1.049 \mathrm{GeV}$.

with electron, photon and pion probes and contains no additional free parameters. RPA and multinucleon knockout have been found to be essential for the description of the data. Our main conclusion is that MiniBooNE data are fully compatible with former determinations of the nucleon axial mass, both using neutrino and electron beams in contrast with several previous analyses. The results also suggest that the neutrino flux could have been underestimated. Besides, we have found that the procedure commonly used to reconstruct the neutrino energy for quasielastic events from the muon angle and energy could be unreliable for a wide region of the phase space, due to the large importance of multinucleon events.

It is clear that experiments on neutrino reactions on complex nuclei have reached a precision level that requires for a quantitative description of sophisticated theoretical approaches. Apart from being important in the study of neutrino physics, these experiments are starting to provide very valuable information on the axial structure of hadrons.

\section{Acknowledgments}

We thank L. Alvarez Ruso, R. Tayloe and G. Zeller for useful discussions. This research was supported by DGI and FEDER funds, under contracts FIS2008-01143/FIS, FIS2006-03438, and the Spanish Consolider-Ingenio 2010 Programme CPAN (CSD2007-00042), by Generalitat Valenciana contract PROMETEO/2009/0090 and by the EU HadronPhysics2 project, grant agreement n. 227431. I.R.S. acknowledges support from the Ministerio de Educación.

[1] C. F. Perdrisat, V. Punjabi and M. Vanderhaeghen, Prog. Part. Nucl. Phys. 59, 694 (2007).

[2] V. Bernard, L. Elouadrhiri and U. G. Meissner, J. Phys. G 28 (2002) R1. 
[3] V. Lyubushkin et al. [NOMAD Collaboration], Eur. Phys. J. C 63 (2009) 355.

[4] A. Bodek, S. Avvakumov, R. Bradford and H. S. Budd, Eur. Phys. J. C 53 (2008) 349.

[5] A. A. Aguilar-Arevalo et al. [MiniBooNE Collaboration], Phys. Rev. D 81 (2010) 092005.

[6] S. Boyd, S. Dytman, E. Hernandez, J. Sobczyk and R. Tacik, AIP Conf. Proc. 1189 (2009) 60.

[7] L. Alvarez-Ruso, arXiv:1012.3871 [nucl-th].

[8] O. Benhar and D. Meloni, Phys. Rev. D 80, 073003 (2009).

[9] O. Benhar, P. Coletti and D. Meloni, Phys. Rev. Lett. 105 (2010) 132301.

[10] C. Juszczak, J. T. Sobczyk and J. Zmuda, Phys. Rev. C 82 (2010) 045502.

[11] A. V. Butkevich, Phys. Rev. C 82 (2010) 055501.

[12] M. Martini, M. Ericson, G. Chanfray and J. Marteau, Phys. Rev. C 80, 065501 (2009).

[13] M. Martini, M. Ericson, G. Chanfray and J. Marteau, Phys. Rev. C 81, 045502 (2010).

[14] J. E. Amaro, M. B. Barbaro, J. A. Caballero, T. W. Donnelly and C. F. Williamson, Phys. Lett. B 696 (2011) 151.

[15] J. Nieves, I. Ruiz Simo and M. J. Vicente Vacas, Phys. Rev. C 83 (2011) 045501.

[16] J. Nieves, J. E. Amaro and M. Valverde, Phys. Rev. C 70 (2004) 055503 [Erratum-ibid. C 72 (2005) 019902].

[17] J. Nieves, M. Valverde and M. J. Vicente Vacas, Phys. Rev. C 73 (2006) 025504.

[18] A. Gil, J. Nieves and E. Oset, Nucl. Phys. A 627 (1997) 543.

[19] E. Oset, H. Toki and W. Weise, Phys. Rept. 83 (1982) 281.

[20] L. L. Salcedo, E. Oset, M. J. Vicente-Vacas and C. Garcia-Recio, Nucl. Phys. A 484 (1988) 557.

[21] R. C. Carrasco and E. Oset, Nucl. Phys. A 536 (1992) 445.

[22] J. Nieves, E. Oset and C. Garcia-Recio, Nucl. Phys. A 554 (1993) 509.

[23] J. Nieves, E. Oset and C. Garcia-Recio, Nucl. Phys. A 554 (1993) 554.

[24] J. E. Amaro, M. B. Barbaro, J. A. Caballero, T. W. Donnelly and J. M. Udias, Phys. Rev. D 84 (2011) 033004.

[25] C. Barbero, G. Lopez Castro and A. Mariano, Phys. Lett. B 664 (2008) 70; A. Mariano, C. Barbero and G. Lopez Castro, Nucl. Phys. A 849 (2011) 218.

[26] G. D'Agostini, Nucl. Instrum. Meth. A 346, 306 (1994).

[27] T. Leitner and U. Mosel, Phys. Rev. C 81 (2010) 064614.

[28] E. Fernandez-Martinez and D. Meloni, Phys. Lett. B 697 (2011) 477. 